\title{
TWO REMARKS ON THE SUITA CONJECTURE
}

\author{
NIKOLAI NIKOLOV
}

\begin{abstract}
It is shown that the weak multidimensional Suita conjecture fails for any bounded non-pseudoconvex domain with $C^{1+\varepsilon_{-}}$ smooth boundary. On the other hand, it is proved that the weak converse to the Suita conjecture holds for any finitely connected planar domain.
\end{abstract}

Let $D$ be a domain in $\mathbb{C}^{n}$. Denote by $K_{D}$ and $A_{D}$ the Bergman kernel on the diagonal and the Azukawa metric of $D$ (cf. [JP]). Let

$$
I_{D}^{A}(z)=\left\{X \in \mathbb{C}^{n}: A_{D}(z ; X)<1\right\}
$$

be the indicatrix of $A_{D}$ at $z \in D$.

Z. Blocki and W. Zwonek have recently proved the following (see [BZ, Theorem 2] and [B2, Theorem 7.5]).

Theorem 1. If $D$ is a pseudoconvex domain in $\mathbb{C}^{n}$, then

$$
K_{D}(z) \geq \frac{1}{\lambda\left(I_{D}^{A}(z)\right)}, \quad z \in D ! 1
$$

Theorem 1 for $n=1$ is known as the Suita conjecture (see $[\mathbf{S}]$ ). The first proof of this conjecture is given in [B1].

Theorem 1 can hold for some bounded non-pseudoconvex domains. To see this, note that if $M$ is a closed pluripolar subset of a domain $D$ in $\mathbb{C}^{n}$, then $K_{D \backslash M}=K_{D}$ and $A_{D \backslash M}=A_{D}$.

On the other hand, our first remark says that even a weaker version of Theorem 1 fails for bounded non-pseudoconvex domains with $C^{1+\varepsilon_{-}}$ smooth boundaries.

Proposition 2. Let $D$ is a bounded non-pseudoconvex domain in $\mathbb{C}^{n}$ with $C^{1+\varepsilon}$-smooth boundary $(\varepsilon>0)$. Then there exists a sequence $\left(z_{j}\right)_{j} \subset D$ such that

$$
\lim _{j \rightarrow \infty} K_{D}\left(z_{j}\right) \lambda\left(I_{D}^{A}\left(z_{j}\right)\right)=0
$$

2010 Mathematics Subject Classification. 32A25, 32F45, 32U35.

Key words and phrases. Suita conjecture, Bergman kernel, Azukawa metric.

${ }^{1}$ If $\lambda\left(I_{D}^{A}(z)\right)=\infty$, then $K_{D}(z)=0$. 
Proof. Since $D$ is non-pseudoconvex, we may find a sequence $\left(z_{j}\right)_{j} \subset D$ approaching $a \in \partial D$ such that

$$
\lim _{j \rightarrow \infty} K_{D}\left(z_{j}\right)<\infty
$$

(otherwise, $\log K_{D}$ would be a plurisubharmonic exhaustion function for $D)$. On the other hand, if $\varepsilon \leq 1$, then, by [DNT, Proposition 2 (i)], there exists a constant $c_{1}>0$ such that

$$
c_{1} A_{D}(z ; X) \geq \frac{\left|X_{N}\right|}{\left(d_{D}(z)\right)^{\frac{\varepsilon}{1+\varepsilon}}}+\|X\|, \quad z \text { near } a,
$$

where $d_{D}(z)=\operatorname{dist}(z, \partial D)$ and $X_{N}$ is the projection of $X$ on the complex normal to $\partial D$ at a point $a^{\prime}$ such that $\left\|z-a^{\prime}\right\|=d_{D}(z)$. Thus, one may find a constant $c_{2}>0$ for which

$$
\lambda\left(I_{D}^{A}(z)\right) \leq c_{2}\left(d_{D}(z)\right)^{\frac{2 \varepsilon}{1+\varepsilon}}, \quad z \text { near } a .
$$

This inequality and (1) imply the wanted result.

Proposition 3. [BZ, Proposition 4] Let $0<r<1$ and $P_{r}=\{z \in \mathbb{C}$ : $r<|z|<1\}$. Then

$$
K_{P_{r}}(\sqrt{r}) \geq-\frac{2 \log r}{\pi^{2}} \cdot \frac{1}{\lambda\left(I_{P_{r}}^{A}(\sqrt{r})\right)} .
$$

So, the converse to the Suita conjecture is not true with any universal constant instead of 1 . However, our second remark says that any finitely connected planar has its own constant.

Proposition 4. For any finitely connected planar domain $D$ there exists a constat $c>0$ such that

$$
K_{D}(z) \leq \frac{c}{\lambda\left(I_{D}^{A}(z)\right)}, \quad z \in D
$$

Proof. It follows by the removable singularity theorem and the uniformization theorem that it is enough to consider the case when $D=$ $\mathbb{D} \backslash E$, where $E$ is a union of disjoint closed discs which belong to the open unit disc $\mathbb{D}$. Since now $\partial D$ is $C^{1}$-smooth, then [JN, Proposition 2] (see also [JP, Lemma 20.3.1]) and the remark at the end of [JN] show that

$$
\lim _{z \rightarrow \partial D} \frac{d_{D}^{2}(z)}{\lambda\left(I_{D}^{A}(z)\right)}=\frac{1}{4 \pi}=\lim _{z \rightarrow \partial D} K_{D}(z) d_{D}^{2}(z) .
$$

Hence

$$
\lim _{z \rightarrow \partial D} K_{D}(z) \lambda\left(I_{D}^{A}(z)\right)=1
$$

which leads to the desired inequality. 


\section{REFERENCES}

[B1] Z. Blocki, Suita conjecture and the Ohsawa-Takegoshi extension theorem, Invent. Math. 193 (2013), 149-158.

[B2] Z. Blocki, Cauchy-Riemann meet Monge-Ampère, Bull. Math. Sci. 4 (2014), 433-480.

[BZ] Z. Blocki, W. Zwonek, Estimates for the Bergman kernel and the multidimensional Suita conjecture, arXiv:1404.7692,

[DNT] N. Q. Dieu, N. Nikolov, P. J. Thomas, Estimates for invariant metrics near non-semipositive boundary points, J. Geom. Anal. 23 (2013), 598-610.

[JN] M. Jarnicki, N. Nikolov, Behavior of the Carathéodory metric near strictly convex boundary points, Univ. Iag. Acta Math. XL (2002), 7-12.

[JP] M. Jarnicki, P. Pflug, Invariant distances and metrics in complex analysis - 2nd extended edition, de Gruyter, 2013.

[S] N. Suita, Capacities and kernels on Riemann surfaces, Arch. Ration. Mech. Anal. 46 (1972), 212-217.

Institute of MATHEMATiCs AND Informatics, Bulgarian ACADEMy OF Sciences, Acad. G. Bonchev Str., Block 8, 1113 Sofia, Bulgaria

E-mail address: nik@math.bas.bg

FACUlty of Information Sciences, State University of Library StudIES AND Information Technologies, 69A, Shipchenski Prohod Str., 1574 SOFiA, Bulgaria 